\title{
A clinically silent respiratory infection with Chlamydophila spp. in calves is associated with airway obstruction and pulmonary inflammation
}

\author{
Julia JAEGER ${ }^{\mathrm{a}, \mathrm{b}}$, Elisabeth LIEBLER-TENORIO ${ }^{\mathrm{a}}$, Nathalie KIRSCHVINK ${ }^{\mathrm{c}}$, \\ Konrad SACHSE ${ }^{\text {a }}$, Petra REINHOLD ${ }^{\text {a* }}$ \\ ${ }^{a}$ Institute of Molecular Pathogenesis in the Friedrich-Loeffler-Institute \\ (Federal Research Institute for Animal Health), Naumburger Str. 96a, 07743 Jena, Germany \\ ${ }^{\mathrm{b}}$ Present address: Universität Leipzig, Veterinärmedizinische Fakultät Ambulatorische und \\ Geburtshilfliche Tierklinik, An den Tierkliniken 29, 04103 Leipzig, Germany \\ ${ }^{c}$ Animal Physiology, Department of Veterinary Medicine, Faculty of Sciences, University of Namur, \\ rue de Bruxelles 61, 5000 Namur, Belgium
}

(Received 12 January 2007; accepted 10 April 2007)

\begin{abstract}
This study was aimed at evaluating functional and inflammatory consequences of persistent chlamydial infections on the respiratory system in clinically inconspicuous calves aged 2-7 months. Thirteen calves persistently infected with Chlamydophila $(C$.) abortus and/or C. pecorum $(\mathrm{Chl}+)$ were compared to 12 calves without chlamydial infections (Chl-). In order to evaluate lung function, 36 non-invasive impulse oscillometry tests were performed per animal within 6 months. The group of chronically infected animals was distinguished by significantly higher peripheral airway resistance (indicating peripheral airway obstruction), significantly higher respiratory rates, and significantly higher minute volumes of ventilation. At the age of seven months, all calves were necropsied, broncho-alveolar lavage fluid (BALF) was obtained ex vivo, and lungs were examined histologically. Significantly higher concentrations of total protein and 8-iso-prostane (8-IP), as well as higher activities of matrix metalloprotease 2 were measured in BALF samples of $\mathrm{Chl}+$ calves. Histologically, markedly activated bronchus-associated lymphoid tissue (BALT) causing partial obstruction of bronchiolar lumina was found in the apical pulmonary lobes of Chl+ calves. Chlamydial DNA was detected in the lung tissue of 7 out of $13 \mathrm{Chl}+$ calves by real-time PCR. In conclusion, respiratory chlamydial infection appeared to be associated with chronic inflammation of the lungs and airways. Despite the lack of clinical symptoms, pulmonary dysfunctions persisted in calves until the age of seven months. Data obtained in this study provide new insight illustrating the impact of nearly ubiquitous subclinical infections on the respiratory system.
\end{abstract}

Chlamydia / Chlamydophila infection / airway obstruction / pulmonary inflammation / calves

\section{INTRODUCTION}

The role of Chlamydia and/or Chlamydophila $(C$.) spp. as respiratory pathogens

\footnotetext{
* Corresponding author: petra.reinhold@ fli.bund.de
}

has been a subject of continuing debate in both human and veterinary medicine. Being obligate intracellular bacteria, chlamydiae are entirely dependent on the host for their replication within cells, where they form characteristic vacuole-like inclusions 
and replicate within an endosome. As a consequence of the distinctive features of the causative agents, a remarkable diversity of clinical manifestations of chlamydioses has been noted. First, chlamydial infections are known to generate acute illness. Even life-threatening situations have been reported in humans who became infected with zoonotic chlamydiae, such as $C$. psittaci originating from birds or C. abortus from small ruminants $[9,12,28$, $54,55]$. Secondly, chlamydial organisms are reported to persist and cause recurrent infections or re-activation of latent infections $[7,11,25,46]$.

In humans, infections with Chlamydophila (formerly Chlamydia) pneumoniae are known to be involved in a variety of clinical outcomes related to the respiratory system. Acute chlamydial infections have been reported to affect upper airways (e.g. pharyngitis, sinusitis, and otitis), central and lower airways (e.g. bronchitis, brochiolitis), as well as lung tissue (pneumonia) $[6,8,10,21,38]$. The phenomenon of chronicity and persistence, however, is thought to be involved in the pathogenesis of chronic inflammatory airway diseases, i.e. human asthma and COPD, and even in pulmonary emphysema or lung cancer [2, $3,14,19,22,26,50,51,56]$.

In cattle, several reports suggest a link between Chlamydophila infections and various disease syndromes, although neither the prevalence nor the economic impact of bovine chlamydial infections can be adequately assessed at present [20]. In traditional veterinary literature, respiratory chlamydial infections in bovines were mostly subsumed under the generic term of 'pneumonia' characterised by fever and depression, nasal secretions, cough, and dyspnoea [47]. Recently an outbreak of acute upper respiratory tract disease was reported in calves aged less than six months [53]. Furthermore, chlamydial infections have been found to be associated with keratoconjunctivitis or polyarthritis, respectively in calves $[27,52]$. In calves from chlamydia-positive dairy herds, the adult cows are the most likely source of infection leading to the presence of $C$. abortus and/or $C$. pecorum in both the intestinal and the respiratory tracts in the first two weeks after birth $[13,53]$. Such infections do not necessarily lead to clinical illness, but may have a serious impact on livestock productivity and farm income ${ }^{1}$. Health effects of subclinical, chronic, or recurrent chlamydial infections in cattle have yet to be defined in veterinary medicine.

This prospective study of the respiratory system in calves was undertaken in order to answer the question whether, even in the absence of clinical signs, there are any consequences for the organ system where chlamydiae reside. To the best of our knowledge, there are no published data unravelling functional consequences of subclinical chlamydial infections in the bovine respiratory system. Consequently, the present study was designed to evaluate the influence of naturally occurring, but clinically inapparent infections with C. abortus and/or C. pecorum on lung function and markers of pulmonary inflammation in conventionally raised calves during postnatal lung maturation until seven months of age.

\section{MATERIALS AND METHODS}

\subsection{Animals}

In this prospective study, 25 conventionally raised calves (Holstein crossbred; 19 female and 6 male) were included and grouped according to their chlamydial carrier status. The latter was defined

\footnotetext{
${ }^{1}$ Kaltenboeck B., Recent advances in the knowledge of animal chlamydial infections, in: Schachter J. et al. (Eds.), Chlamydial Infections, Proc. 11th Int. Symposium on Human Chlamydial Infection, Niagara-on-theLake, Canada, June 2006, pp. 399-408.
} 
in vivo on the basis of diagnostic examination of 23 swabs per animal (nasal, rectal, and conjunctival) taken consecutively at 3-4 week intervals throughout the study. This monthly testing, as well as ex vivo tissue examination, revealed persistent or frequently recurring infections with $C$. pecorum and $C$. abortus in Group 'Chl+' $(n=13)$, but not in Group 'Chl-' $(n=12)$. For chlamydia detection, both a nested PCR procedure targeting the ompA gene [36], and a DNA microarray assay [37] were used. Serology (complement fixation test) was conducted to confirm chlamydial infections in Group Chl+. No epidemiologically important co-infections were identified as confounding factors considering the following agents: Salmonella, Mycoplasma, Pasteurella and Mannheimia spp., BHV-1 (bovine herpes virus 1), BVDV (bovine virus diarrhoea/mucosal disease virus), BRSV (bovine respiratory syncytial virus), PI-3 (parainfluenza 3 virus), and adenovirus type 3 . Further details characterising these 25 calves have been described elsewhere [33].

The animals were reared under standardised conditions (room climate: 18 to $20{ }^{\circ} \mathrm{C}, 65 \%$ relative humidity) and in accordance with international guidelines for animal welfare. Nutrition contained commercial milk replacers and coarse meal. Water and hay were supplied ad libitum. None of the given feed contained antibiotics.

After a quarantine period of at least two weeks and confirmation of a clinically normal status, all 25 animals were included in the study. For each animal, the observation period lasted from the 2nd until 7 th month of age. Within this period, each calf underwent daily clinical examination and repeated lung function testing. At the age of seven months, the animals were euthanised, broncho-alveolar lavage fluid was obtained ex vivo, and the lungs were examined histologically. All phases of the in vivo study were non-invasive, and ethical approval was granted by the Institutional Commission for the Protection of Animals.

\subsection{Pulmonary function tests}

For lung function testing, the impulse oscillometry system ("MasterScreenIOS", VIASYS Healthcare, Hoechberg, Germany) was used as described by Smith et al. [45]. This non-invasive technique was previously validated for conscious calves [29-31]. Calves were adapted to the system using a tightly fitting facemask of appropriate size depending on the animal's head. Each lung function test was performed in the conscious calf during spontaneous breathing.

Starting at the age of two months, six tests were performed per calf and month (three consecutive tests per day and animal on two consecutive days). The duration of one test was $60 \mathrm{~s}$ while 3 test impulses were generated per second (leading to 180 independent test results per minute). The sampling rate was set at $200 \mathrm{~Hz}$ (period between two sampling points of $5 \mathrm{~ms}$ ), selecting 32 sampling points after each impulse. The following variables of ventilation were analysed: respiratory rate $(\mathrm{RR})$; tidal volume $(\mathrm{Vt})$; volume of minute ventilation (Vmin); both, tidal volume and minute volume related per $\mathrm{kg}$ body weight $(\mathrm{Vt} / \mathrm{kg}, \mathrm{Vmin} / \mathrm{kg})$. In order to evaluate respiratory mechanics, respiratory resistance $(\mathrm{R})$ and respiratory reactance $(\mathrm{X})$ were analysed in dependence on frequency [45]. Recalculation of original IOS-data using the software FAMOS (Fast Analysis and Monitoring of Signals; imc Mess-Systeme GmbH, Berlin, Germany) enabled us to calculate $\mathrm{R}$ and $\mathrm{X}$ for each frequency within the frequency range of 1 to $10 \mathrm{~Hz}$, and separated for inspiration and expiration $(\mathrm{R}$ in $1 \mathrm{~Hz} \ldots \mathrm{R}$ in $10 \mathrm{~Hz}$; $\mathrm{R} \operatorname{ex}_{1 \mathrm{~Hz}} \ldots \mathrm{R} \mathrm{ex}_{10 \mathrm{~Hz}} ; \mathrm{X}$ in ${ }_{1 \mathrm{~Hz}} \ldots \mathrm{X}$ in ${ }_{10 \mathrm{~Hz}}$; $\mathrm{X}$ ex ${ }_{1 \mathrm{~Hz}} \ldots \mathrm{X}$ ex $\left.{ }_{10 \mathrm{~Hz}}\right)$. In addition, the IOS 
software allowed calculating of proximal airway resistance ( $\mathrm{R}$ prox) and distal airway resistance ( $\mathrm{R}$ dist). The results of all six lung function measurements per animal and month were averaged and these average values were used for further analysis. In order to take the influence of body weight increase on variables of lung function into account, body weight was always measured before monthly lung function testing.

\subsection{Necropsy and collection of serum, BALF, and tissue samples}

At the end of the study, all animals were euthanised and necropsied. Immediately before euthanasia, venous blood was collected in $7.5 \mathrm{~mL}$-syringes (S-Monovette ${ }^{\circledR}$, Sarstedt AG \& Co, Nuembrecht, Germany) for serum production. Under conditions of deep anaesthesia (thiopental sodium, $1.5 \mathrm{~g}$ per $100 \mathrm{~kg}$ body weight, intravenously, Trapanal ${ }^{\circledR}$, Altana Pharma, Konstanz, Germany), the trachea was exposed and large clamps were placed distal to the larynx to prevent contamination of the airways by blood or gastric contents. Subsequently, the animals were exsanguinated and the lung was removed. Macroscopic lesions of the lung and the other organs were recorded.

Broncho-alveolar lavage (BAL) was performed using glass syringes and a catheter was instilled through the trachea into either the left or right basal lobe. Five subsequent washes using $10 \mathrm{~mL}$ for each installation (in total $50 \mathrm{~mL}$ ) of Aqua bidest. were performed. After immediate aspiration, the recovered BAL fluid (BALF) was about $50 \%$ and did not differ significantly between groups. The supernatant of BALF was harvested by centrifugation $\left(5{ }^{\circ} \mathrm{C}\right.$, $3939 \mathrm{~g}, 20 \mathrm{~min}$ ).

Tissue samples were collected from six lung sites, i.e. left and right cranial portion of the apical lobe, left and right cau- dal portion of the apical lobe, left and right basal lobe, and the tracheobronchial and pulmonary lymph nodes. Aliquots of each sample were used for bacteriological, molecular and histological examination.

\subsection{Determination of matrix metalloprotease activity in BALF and serum}

BALF supernatant and serum samples were kept at $-20{ }^{\circ} \mathrm{C}$ until analysis. The activity of matrix metalloprotease 2 (MMP-2), and MMP-9 was measured by gelatin zymography [15]. Standards of MMP-9 (human pro-enzyme MMP-9; Oncogene, San Diego, CA, USA), of MMP-2 (human pro-enzyme MMP-2; Oncogene, San Diego, CA, USA) and BALF and serum samples diluted in non-reducing sample buffer were loaded onto $6 \%$ acrylamide SDS gels containing porcine skin gelatine $(0.1 \%$; SigmaAldrich, Bornem, Belgium). After electrophoresis, the gels were washed twice in Triton X-100 2\% for $30 \mathrm{~min}$ and incubated at $37{ }^{\circ} \mathrm{C}$ for $48 \mathrm{~h}$ (BALF samples) and $24 \mathrm{~h}$ (serum samples) in an activation buffer containing $50 \mathrm{mM}$ Tris $\mathrm{HCl}(\mathrm{pH} 7.5)$ and $10 \mathrm{mM} \mathrm{CaCl}_{2}$. Following incubation, the gels were rinsed and stained for $30 \mathrm{~min}$ with Coomassie blue and destained for $\sim 2 \mathrm{~h}$ in a solution of $10 \%$ acetic acid and $20 \%$ methanol (v/v). Gelatinolytic activity appeared as unstained zones against a blue background. The gels were scanned (Epson Perfection 2450 Photo; Seiko Epson Corp., Nagano, Japan) and converted to numeric images for quantification using the Scion imaging analysis programme (Scion Corporation, Frederick, Maryland, USA). Activated forms of MMP-2 and MMP-9 were rarely apparent and quantified simultaneously with pro-forms. The results were expressed as average arbitrary units (AU) corresponding to pixel density $\times \mathrm{mm}^{2}$ for the bands of proteolysis. 


\subsection{Determination of 8-isoprostane concentration and protein concentration}

In both BALF and serum samples, 8iso-prostane (8-IP) was analysed by a commercially available enzyme-immunoassay (EIA; Cayman Chemical Company, Ann Arbor, Michigan, USA), and the results were verified by means of LC/MS using an external 8-IP solution. The detection limit was $\geqslant 3.9 \mathrm{pg} / \mathrm{mL}$

Total protein in BALF samples was measured colorimetrically using a Pierce Micro BCA(TM) Reagent Kit (Pierce, Rockford, IL, USA) in duplicate. The sensitivity of this method was $\geqslant 0.5 \mu \mathrm{g} / \mathrm{mL}$.

\subsection{Histopathology, immunohistology for chlamydial antigen and PCR for detection of chlamydial DNA in pulmonary tissue}

One part of each sample was immersion fixed in neutral buffered formalin, embedded in paraffin, sectioned and stained with haematoxylin and eosin (H\&E) for histological evaluation.

The other sample was snap frozen at $-70{ }^{\circ} \mathrm{C}$ for immunohistochemistry. For this, $5 \mu \mathrm{m}$ thick cryostat sections were prepared and fixed in $4 \%$ formalin with $67 \mu \mathrm{mol} / \mathrm{L}$ calcium chloride ( $\mathrm{pH}$ 7.2) for $10 \mathrm{~min}$ at $4{ }^{\circ} \mathrm{C}$. Chlamydial antigen was detected by the indirect immunoperoxidase method using a monoclonal antibody against chlamydial LPS (ACI-P, Progen, Heidelberg, Germany) and a peroxidaselabelled anti-mouse Ig (NA 931, GE Healthcare Europe GmbH, Freiburg, Germany) as the secondary antibody. Right and left cranial and caudal apical lung lobes from all calves of Group Chl+ were examined for chlamydial antigen. Porcine lung containing chlamydiae was used as a positive control.

For detection of chlamydiae by PCR, about $20 \mu \mathrm{m}$ thick slices from frozen tissue that had been used for immunohistology in the $\mathrm{Chl}+$ calves, and frozen tissue from the respective lung lobes of the Chl- calves were pooled for each calf in Eppendorf tubes. Tissue samples were DNA extracted using the High Pure PCR Template Preparation Kit (Roche, Mannheim, Germany) according to the manufacturer's instructions. Chlamydial DNA was detected by 23S rRNA real-time PCR as described previously [5]. Each sample was examined in quadruplicate.

\subsection{Statistical analyses}

Statistical analyses were used to clarify whether the presence of Chlamydophila spp. had a significant influence on pulmonary functions as evaluated by impulse oscillometry measurements, peripheral blood parameters, inflammatory mediators, or clinical symptoms. Therefore, SPPS (Version 12.0 for Windows; SPSS Inc., Chicago, Illinois, USA) and Statgraphics Plus (Version 4.0 for Windows; Manugistics Inc., Rockville, Maryland, USA) were explored.

Normally distributed data are given as mean \pm standard deviation (SD), whereas data of non-normal or unknown distribution are always presented as median, minimum and maximum. For statistical analysis of multiple data with normal distribution, multifactorial analysis of variance (ANOVA) was used (multiple range test based on least significant difference). To compare two unpaired samples (i.e. differences between two groups at one time point), the unpaired $t$ test (comparison of means) was used for normally distributed data. The $W$ test according to Mann-Whitney (Wilcoxon) was used to compare medians of two unpaired samples with non-normal or unknown distribution. For statistical significance, probability levels are given with the results. Since the $P$-value is equal to or less than 0.05 , there 

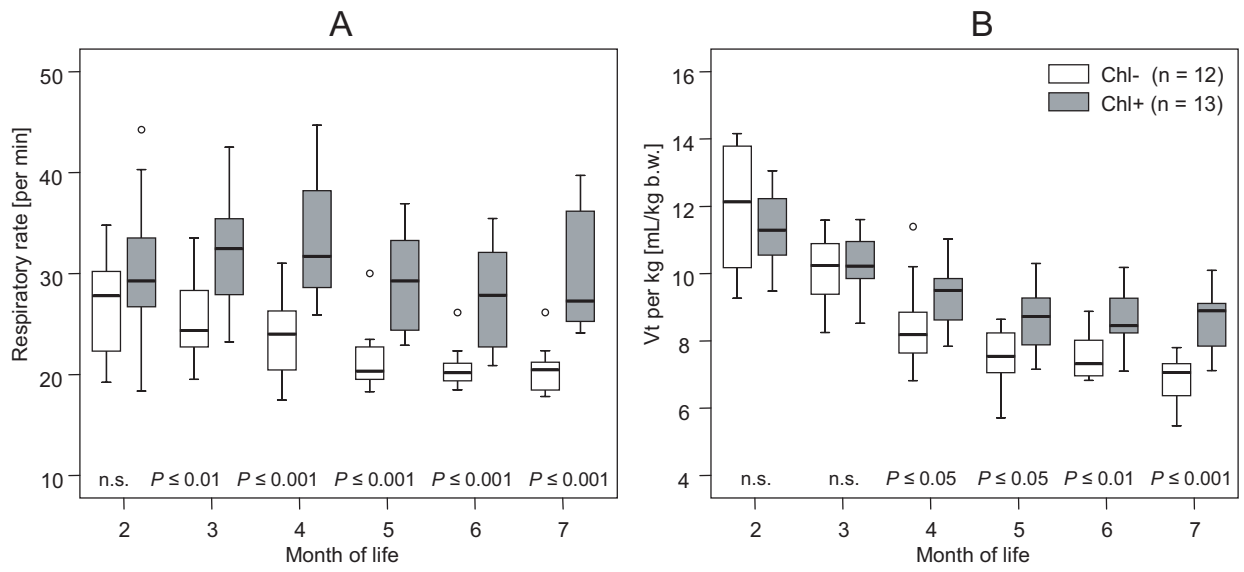

Figure 1. Respiratory rate (A) and tidal volume per kg body weight $(\mathbf{B})$ in calves aged 2 to 7 months. Chl-: Calves without chlamydial infections $(n=12)$; Chl+: calves with clinically latent chlamydial infections $(n=13)$; Box-and-Whisker Plot represents median value, 25\%- and 75\% percentiles (box), range, and outlier values (o). $P$-values indicate significant differences between groups at given time points ( $W$ test).

is a statistically significant difference at the $95.0 \%$ confidence level.

\section{RESULTS}

\subsection{Parameters of ventilation and respiratory mechanics}

\subsubsection{Respiratory rate, tidal volume and minute ventilation}

While respiratory rate decreased significantly in calves without chlamydial infection over time (ANOVA, LSD, $P \leqslant 0.01$ ), it did not change significantly in calves with Chlamydophila infections (Chl+). Consequently, in calves aged 3 to 7 months, RR was significantly higher in the $\mathrm{Chl}+$ Group compared to the Chl- Group (Fig. 1A).

In order to eliminate the influence of different body weights between groups on lung volumes, both tidal volume and minute volume were compared after calculation per $\mathrm{kg}$ body weight (b.w.). At the age of two months, neither tidal nor minute volume per $\mathrm{kg}$ b.w. was significantly different between groups. In calves aged three months and older, the volume of minute ventilation related to body weight was significantly higher in the Chl+ Group compared to the Chl- Group (Tab. I). Tidal volume per $\mathrm{kg}$ b.w. was significantly higher in calves of the Chl+ Group aged four months and older (Fig. 1B).

\subsubsection{Respiratory impedance (resistance and reactance, each at inspiration and expiration) and coherence}

Physiologically, respiratory resistance decreases while respiratory reactance increases with increasing age and body weight. To identify possible differences in respiratory mechanics between groups, resistances as well as reactances were calculated per kg b.w. and compared at each time point. For two time points, i.e. at the beginning and end of the study, the numerical results of expiratory resistance and reactance (both related per kg b.w.) are given in Table II. The frequency dependence of 
Table I. Volume of minute ventilation per kg body weight (Vmin in $\mathrm{mL} / \mathrm{kg}$ ) in calves without (Chl-, $n=12)$ und calves with chlamydial infections $(\mathrm{Chl}+, n=13)$ aged 2 to 7 months.

\begin{tabular}{|c|c|c|c|c|c|}
\hline Month of life & Group & Median & Minimum & Maximum & $W$ test \\
\hline \multirow[t]{2}{*}{2} & Chl- & 311.8 & 267.7 & 401.6 & \multirow{2}{*}{ n.s. } \\
\hline & Chl+ & 345.0 & 232.0 & 466.9 & \\
\hline \multirow[t]{2}{*}{3} & Chl- & 256.2 & 223.1 & 276.4 & \multirow{2}{*}{$P \leqslant 0.00$} \\
\hline & Chl+ & 317.4 & 259.7 & 438.4 & \\
\hline \multirow[t]{2}{*}{4} & Chl- & 195.7 & 158.4 & 221.9 & \multirow{2}{*}{$P \leqslant 0.001$} \\
\hline & Chl+ & 317.0 & 245.9 & 400.2 & \\
\hline \multirow[t]{2}{*}{5} & Chl- & 156.0 & 145.6 & 181.7 & \multirow{2}{*}{$P \leqslant 0.001$} \\
\hline & $\mathrm{Chl+}$ & 244.4 & 212.5 & 298.1 & \\
\hline \multirow[t]{2}{*}{6} & Chl- & 151.7 & 132.5 & 180.1 & \multirow{2}{*}{$P \leqslant 0.001$} \\
\hline & Chl+ & 241.4 & 191.0 & 295.8 & \\
\hline \multirow[t]{2}{*}{7} & Chl- & 140.4 & 123.1 & 148.2 & \multirow{2}{*}{$P \leqslant 0.001$} \\
\hline & Chl+ & 241.7 & 210.3 & 334.8 & \\
\hline
\end{tabular}

n.s.: No significant difference $(P \geqslant 0.05)$.

A

Expiratory respiratory impedance, 2 months of life

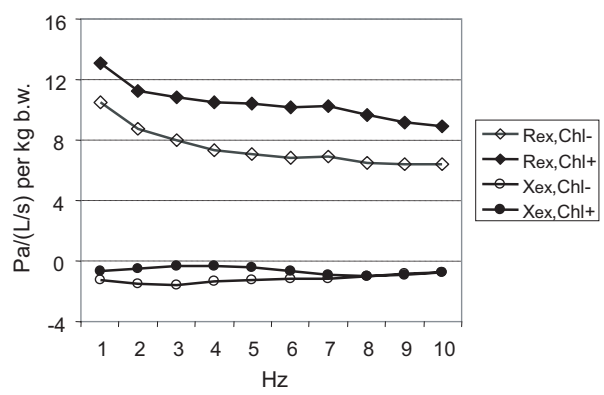

B

Expiratory respiratory impedance, 7 months of life

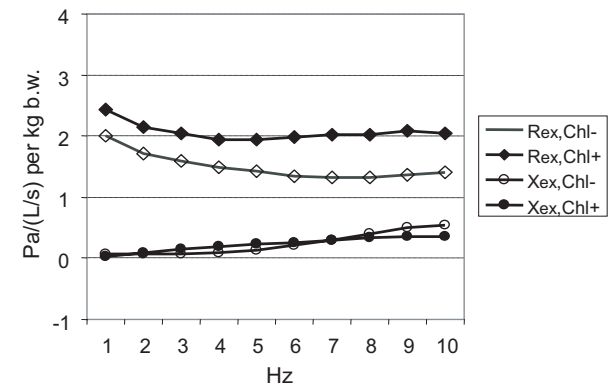

Figure 2. Respiratory impedance during expiration (expressed as respiratory resistance ( $\mathrm{R}$ ex) and respiratory reactance (X ex) per kg. body weight) in calves aged 2 months $(\mathbf{A})$ and 7 months $(\mathbf{B})$. Chl-: Calves without chlamydial infections $(n=12)$; Chl+: calves with clinically latent chlamydial infections $(n=13)$.

$\mathrm{R}$ ex and $\mathrm{X}$ ex as obtained at the age of 2 months and at the age of seven months is shown in Figure 2.

Already at the age of two months, expiratory resistance in calves with chlamydial infections $(\mathrm{Chl}+)$ was markedly higher than in Chl-calves, and this difference between groups remained significant during the course of the study. Consequently, even at the age of seven months, calves of the Chl+ Group showed a much higher expiratory resistance per $\mathrm{kg}$ b.w. than calves of the Chl-Group. As also shown in Figure 2, respiratory reactance values per $\mathrm{kg}$ b.w. were comparable between groups despite statistical analysis resulting in a few significant differences at individual frequencies (Tab. II). 


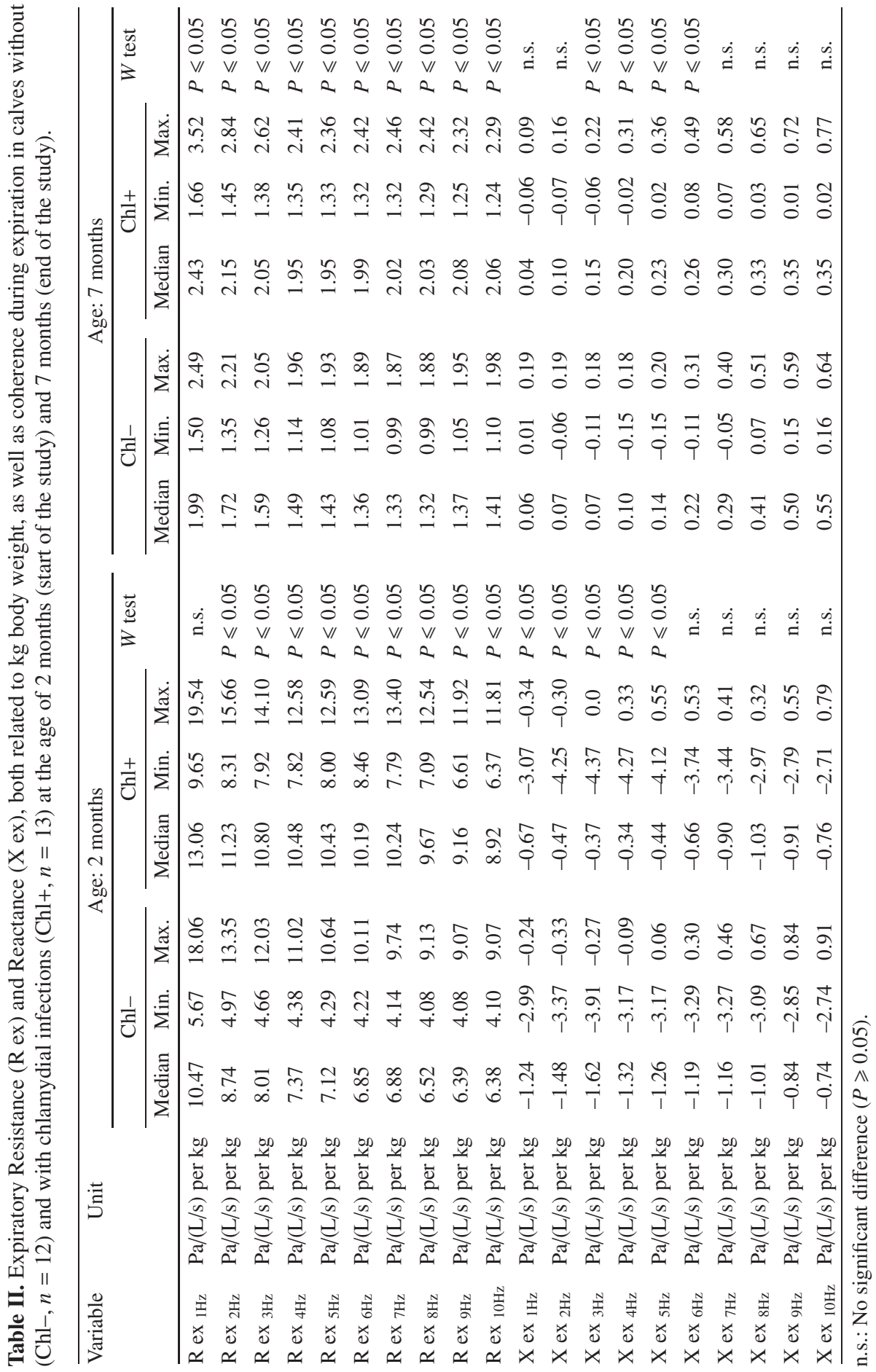


Table III. Markers of inflammation measured in broncho-alveolar lavage fluids (BALF) and in blood sera of 25 clinically normal calves aged 7 months without (Chl-) and with (Chl+) chlamydial infections.

\begin{tabular}{|c|c|c|c|c|c|c|c|c|c|c|c|}
\hline \multirow[t]{2}{*}{ Medium } & \multirow[t]{2}{*}{ Variable } & \multirow[t]{2}{*}{ Unit } & \multicolumn{4}{|c|}{ Chl- } & \multicolumn{4}{|c|}{$\mathrm{Chl+}$} & \multirow[t]{2}{*}{$W$ test } \\
\hline & & & $n$ & Median & Min. & Max. & $n$ & Median & Min. & Max. & \\
\hline \multirow[t]{4}{*}{ BALF $^{\#}$} & total protein & $\mu \mathrm{g} / \mathrm{mL}$ & 12 & 340.0 & 148.0 & 705.0 & 13 & 610.0 & 391.0 & 2205.0 & $P \leqslant 0.01$ \\
\hline & 8-IP & $\mathrm{pg} / \mathrm{mL}$ & 12 & 104.2 & 26.1 & 647.0 & 13 & 340.0 & 64.7 & 908.0 & $P \leqslant 0.05$ \\
\hline & MMP-2 & $\mathrm{AU}$ & 12 & 403.0 & 177.0 & 954.0 & $12 *$ & 836.5 & 453.0 & 1591.0 & $P \leqslant 0.001$ \\
\hline & MMP-9 & $\mathrm{AU}$ & 12 & 303.5 & 143.0 & 811.0 & $12^{*}$ & 506.0 & 38.0 & 1870.0 & n.s. \\
\hline \multirow[t]{3}{*}{ Blood serum ${ }^{\#}$} & 8-IP & $\mathrm{pg} / \mathrm{mL}$ & 12 & 35.0 & 17.6 & 152.1 & 13 & 43.9 & 19.6 & 123.2 & n.s. \\
\hline & MMP-2 & $\mathrm{AU}$ & 12 & 2908.0 & 1204.0 & 3785.0 & 13 & 2209.0 & 624.0 & 3071.0 & n.s. \\
\hline & MMP-9 & $\mathrm{AU}$ & 12 & 1574.5 & 727.0 & 1941.0 & 13 & 1251.0 & 611.0 & 1814.0 & n.s. \\
\hline
\end{tabular}

\#Blood and BALF were obtained at the same day in calves aged 7 months (blood in vivo before euthanisation; BALF samples ex vivo immediately after euthanisation). AU: arbitrary units. * One BALF sample was not possible to be analysed. n.s. $=$ No significant difference between groups $(P>0.05)$.

\subsubsection{Model-derived parameters of respiratory mechanics}

Both resistance of proximal airways and distal airway resistance are shown in Figure 3. Resistance of proximal airways was not significantly different between groups at any time point. At the beginning of the study, resistance of distal airways was comparable between groups. In calves older than three months, however, $\mathrm{R}$ dist was significantly higher in calves with chlamydial infections $(\mathrm{Chl}+)$ compared to calves of the Chl-Group.

\subsection{Markers of inflammation}

At the end of the study, 8-IP, MMP-2 and MMP-9 were analysed in BALF and corresponding blood serum. In addition, the concentration of total protein was measured in BALF. As shown in Table III, all markers of inflammation were higher in BALF samples of the Chl+ Group compared to those of the Chl- Group. For total protein, 8-IP, and MMP-2 the difference between groups was statistically secured. In contrast to BALF, no significant dif- ferences were seen for 8-IP, MMP-2, or MMP-9 in blood samples.

\subsection{Pulmonary lesions}

\subsubsection{Gross lesions}

Macroscopic examination revealed mild lesions in the respiratory tract in both groups of calves. In the Chl- Group, circumscribed chronic adhesive pleuritis was found in five calves predominantly affecting the right cranial apical lobes. One calf had a chronic indurative bronchopneumonia, and two calves had foci of atelectasis in the lung. In the Chl+ Group, purulent bronchopneumonia occurred in two calves and foci of atelectasis in nine calves predominantly affecting the apical lobes. Chronic adhesive pleuritis was noted in only one calf of this group. Using a scoring system between 0 and 3 ( $0=$ no macroscopic lesions, $1=$ atelectasis, $2=$ pneumonic lesions of small extent, $3=$ pneumonic lesions of large extent), a score of $0.9 \pm 0.7$ was observed in the ChlGroup versus $1.4 \pm 1.0$ in the Chl+ Group (means $\pm \mathrm{SD}$, no statistically significant difference between means, $t$ test). 

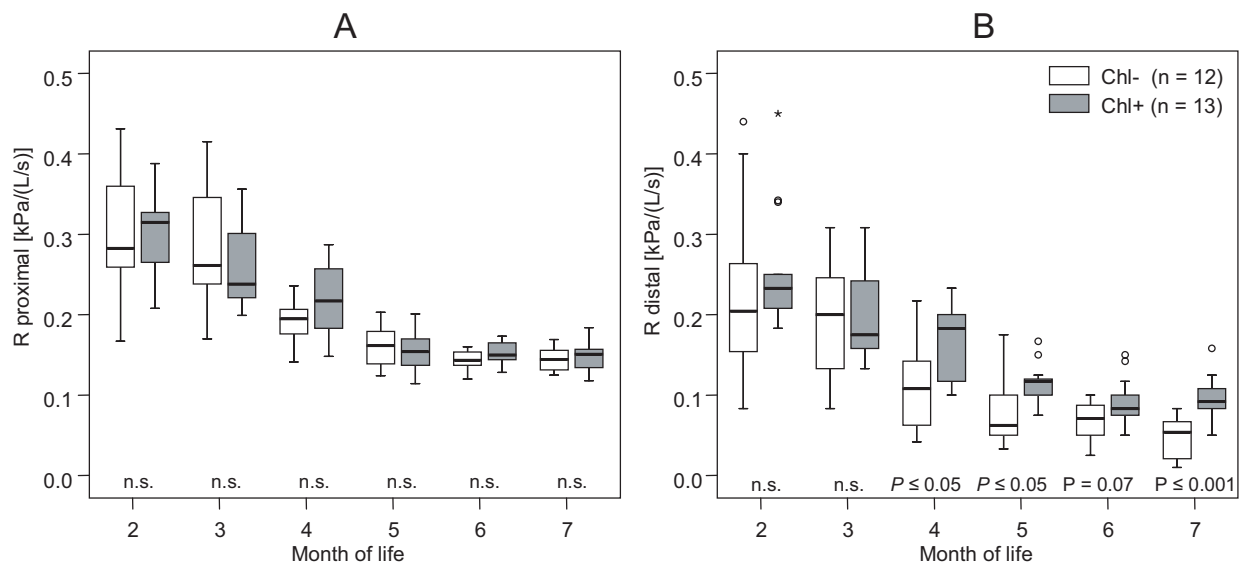

Figure 3. Proximal (A) and distal (B) airway resistance in calves aged 2 to 7 months. Chl-: calves without chlamydial infections $(n=12)$; Chl+: calves with clinically latent chlamydial infections ( $n=13$ ); Box-and-Whisker Plot represents median value, $25 \%$ - and $75 \%$ percentiles (box), range, and outlier values (o). $P$-values indicate significant differences between groups at given time points $(W$ test $)$.

\subsubsection{Histological lesions}

In all calves of the Chl- Group, mild multifocal thickening of the interalveolar septae by lymphocellular infiltrates was observed in different lung lobes. Bronchusassociated lymphoid tissue (BALT) was present in the walls of or close to bronchi, bronchioli and blood vessels (Fig. 4a). Five calves had few and inactive BALT follicles, 2 calves had moderate numbers of BALT follicles, and 5 calves had many BALT follicles frequently containing germinal centres. The majority of BALT follicles was rather small (Fig. 4a), and narrowing of bronchial or bronchiolar lumina by BALT was rarely observed. The chronic indurative bronchopneumonia in the right cranial portion of the apical lobe of one calf was confirmed.

In both groups, comparable changes were seen in the alveoli and interalveolar septae. Differences were observed in BALT and bronchiolar and bronchial morphology. In 12 of the 13 calves of the Chl+ Group, high numbers of enlarged lymphoid follicles were present that protruded into bronchial and bronchiolar lumina, causing a loss of the mucosal folding and partial obstruction. Obstruction was especially marked when cuffing occurred with lymphoid follicles present along the entire circumference of bronchioli (Fig. 4b). In nine calves, the adjacent pulmonary tissue was atelectatic. These changes were particularly frequent in the right cranial apical lobes and in the left caudal apical lobes, but affected more than one lobe in most calves. The bronchial and bronchiolar epithelium covering protruding lymphoid follicles was hyperplastic (Fig. 4b). There was a marked transcytosis of neutrophils in these areas and exudate containing intact neutrophils and their fragments was present in bronchial and bronchiolar lumina (Fig. 4b).

\subsection{Immunohistology for chlamydial antigen and PCR for chlamydial DNA in pulmonary tissue}

There was no specific staining for chlamydial antigen in cryostat sections of 
a

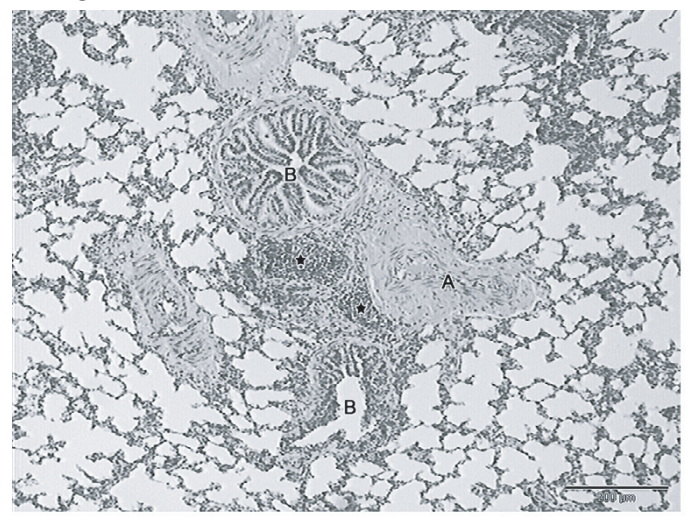

b

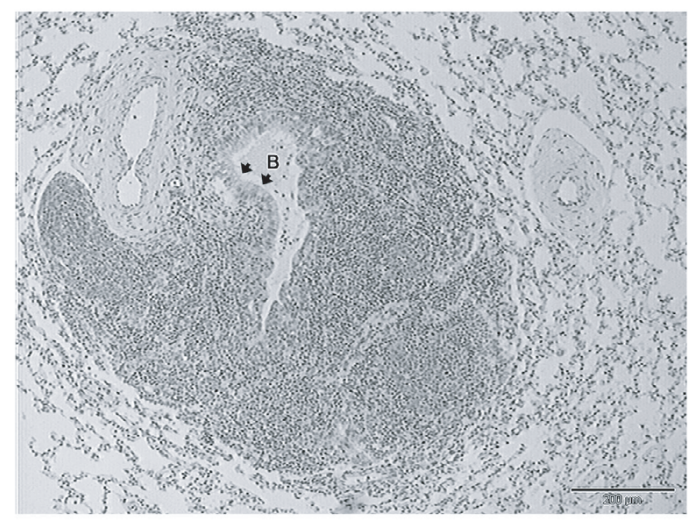

Figure 4. Comparison of pulmonary morphology in Chl-(A) and Chl+ (B) calves (Paraffin sections, H\&E stain, bars $=200 \mu \mathrm{m})$. a. Two small lymphoid follicles $(*)$ close to bronchioles $(B)$ and an arteriole $(A)$ in a Chl- calf (No. 003, left caudal lobe). Note the markedly folded mucosa of the bronchioles. b. Large activated lymphoid follicles with germinal centres completely surrounding a bronchiole (B) ("cuffing") in a Chl+ calf (No. 471, right cranial apical lobe). There is loss of mucosal folding. The bronchiolar lumen is partially obstructed by protruding lymphoid follicles. The bronchiolar epithelium is hyperplastic (arrowheads). Neutrophils and exudates are present in the lumen.

the Chl+ calves. The pulmonary sections examined frequently contained areas of enlarged BALT and hyperplastic bronchioles with partial obstruction. Using real-time PCR, chlamydial DNA was detected in the pooled samples of pulmonary tissue in 7 out of $13 \mathrm{Chl}+$ calves, whereas all samples from Chl-calves were negative.

\section{DISCUSSION}

With the availability of molecular diagnostic tools, particularly PCR, the presence of chlamydiae was frequently detected in animals without acute clinical symptoms ${ }^{1}$. These findings might either be a consequence of persistent infection or repeated 
re-infections resulting in subclinical outcome. Subclinical chlamydial infection has rarely been addressed in veterinary literature. The question arises whether there are any functional consequences on the organ systems infected with chlamydiae in apparently healthy animals. To the best of our knowledge, this is the first study evaluating the functional consequences of chlamydia-host interactions within the bovine respiratory system in the absence of acute clinical illness. Data obtained in this study may help to understand the role of Chlamydophila spp. in the pathogenesis of chronic airway diseases in both humans and animals.

\subsection{Pulmonary function}

Variables of ventilation and respiratory mechanics were evaluated non-invasively and during spontaneous breathing using the impulse oscillometry system (IOS). This technique is based on the principles of the forced oscillation technique and allows frequency-dependent analysis of both $\mathrm{R}$ and $\mathrm{X}$, each separated for inspiration and expiration. While $\mathrm{R}$ reflects resistive properties within the airways, $\mathrm{X}$ is mainly determined by capacitive components of the respiratory system becoming more negative in case of reduced lung compliance. Using a seven-element electrical model of the respiratory system introduced by Mead [23], the resistance of proximal airways ( $\mathrm{R}$ prox) and the resistance of distal airways ( $R$ dist) could be differentiated additionally.

Compared to calves without chlamydial infections, a significantly increased respiratory resistance during expiration was the most striking finding with respect to respiratory mechanics. This phenomenon was already present at the beginning of the study (when calves were aged approximately two months) and was maintained until the end of the study, i.e. until the age of seven months. Since the fre- quency range explored in this study for $\mathrm{R}$ ( 1 to $10 \mathrm{~Hz}$ ) is sensitive to the peripheral airways, data indicate peripheral airway obstruction in Chl+ calves. These data confirm results obtained in a model of experimental respiratory infections with chlamydiae in pigs, where respiratory chlamydial infection also resulted in significant distal airway obstruction [32], and are in agreement with lung function results obtained in children with bronchial C. pneumoniae infection [39].

Since chlamydiae tend to cause chronic infections, a progressive course of functional disturbances is likely, and indeed, obstructive changes became stronger during the course of the study, which is underlined by an increasing discrimination between groups with respect to distal airway resistance (Fig. 3B). According to ten Brinke et al. [49], a C. pneumoniae infection in humans may promote the development of persistent airflow limitation leading to a continuous decline in lung function, and this hypothesis has been confirmed by results in calves aged seven months in comparison to earlier age by the present study. Morphologically, airflow limitation was likely caused by the proliferation of bronchus-associated lymphoid tissue surrounding small bronchi and bronchiolar airway generations. The lymphoid tissue may narrow the airways by external compression, induce partial obstruction by protrusion into the airway lumina and create secondary obstructive changes in the form of intrabronchiolar neutrophils and exudate. The lack of mucosal folding due to the massive infiltration of lymphoid cells in the submucosa indicates reduced elastic dilatation and contraction of the affected airways during breathing. Increased numbers of lymphoid follicles have been reported as a common phenomenon in pulmonary infection of calves and were interpreted as a potential for local inflammation to induce lymphoid tissue within airways [1]. In the present case, 
the increase in number and the hyperplasia were so severe that they caused functional changes.

Reactance data as shown in Figure 2 did not differ markedly despite differences between groups reaching the level of significance at a few frequencies. Higher reactance values in the $\mathrm{Chl}+$ Group were in accordance with significantly higher tidal volumes indicating a higher inflation of the lung during inspiration and excluding decreases in lung compliance or restrictions, respectively. Furthermore, upper or central airways were not affected functionally as presented by similar data for proximal airway resistance (Fig. 3A).

The pattern of breathing as characterised by variables of ventilation (tidal volume, respiratory rate, and volume of minute ventilation) may adapt to a certain amount of disorders in respiratory mechanics in order to fulfil the requirements of the total organism with respect to oxygen supply. At the beginning of the study (calves were aged two months), neither respiratory rates nor tidal or minute volumes were significantly different between both groups. In contrast, significantly higher respiratory rates as well as tidal volumes per kg body weight (and consequently a significantly higher ventilation per minute) were measured in calves older than three months having chlamydial infections $(\mathrm{Chl}+)$ compared to calves of the Chl- Group at the same age. In the absence of restrictive ventilatory disorders, higher tidal volumes might represent compensatory hyperventilation in the presence of inhomogeneities in lung ventilation that are typical in the bovine lung when obstructions occur. Since cattle lack collateral airways [24], peripheral airway obstruction easily leads to ventilatory asynchronisms presented by both atelectases (as seen in the present investigation) and hyperinflated areas [34]. Consequently, a higher volume of minute ventilation in $\mathrm{Chl}+$ calves might indicate less effective alveolar ventilation in the obstructive lung, and result in an increase of the specific ventilation, i.e. raise in Vmin in relation to oxygen intake.

Furthermore, significantly increased minute volumes of ventilation (related to body weight) in Chl+ calves could be caused by an increased metabolic rate that would require more oxygen. This hypothesis is underpinned by the detection of significantly higher, but subfebrile, body temperatures in calves with clinically inapparent chlamydial infections [33]. In addition, a higher load of breathing is present in obstructive areas of the lung leading to intensive work of respiratory muscles. An intensive muscle activity to overcome higher airflow resistances requires more oxygen, too, and this will finally also contribute to an increase of Vmin.

\subsection{Pulmonary inflammation}

Regarding markers of inflammation, the present study revealed a significant increase of total protein and 8-IP concentration in BALF, as well as an increased activity of BALF MMP-2. Systemic repercussions of the pulmonary inflammatory process on serum 8-IP and MMP-2 and MMP-9 activities were not significant. These results indicate that the neutrophilic inflammatory response induced by chlamydial infection was associated with pulmonary oxidative stress (8-IP) and increased MMP-2 activity.

Oxidative stress has been evidenced in children with upper airway infection with $C$. pneumoniae. Significantly higher concentrations of leukotriene B4 and 8-IP in exhaled breath condensate samples were found and indicated neutrophilic inflammation and oxidative stress in the lower airways, whereas cysteinyl leukotriens (LT CDE4), which are likely involved in airway smooth muscle contraction, microvascular leakage and mucous hypersecretion, were not different [41]. Chlamydial infections have been shown to 
be inversely correlated with allergic inflammation in human patients [39, 41]. The results obtained in $\mathrm{Chl}+$ calves also suggest the absence of allergic inflammatory processes.

Our results were further in line with earlier studies showing that MMP-2 and MMP-9 activity might be detected in BALF of healthy calves [16]. In a study investigating MMP-2 and MMP-9 activity in tracheobronchial lavage fluid of calves infected with Pasteurella multocida and Mycoplasma bovirhinis, a significant increase of active MMP-2 and active MMP-9 was found [44]. In our study, MMP-proforms and MMP-active forms were not differentiated for two reasons: only a few Chl+ animals showed active forms and precise quantification of both forms was impossible because a sufficient separation of both bands could not be accomplished. Interestingly, only MMP-2 activity was significantly increased in Chl+ calves, whereas MMP-9 activity did not reach significance. MMP-9 has been shown to be secreted by alveolar neutrophils, macrophages and epithelial cells, whereas MMP-2 was also reported to be secreted by fibroblasts. Increased MMP-2 activity due to persistent neutrophilic inflammation as seen in $\mathrm{Chl}+$ calves and an important fibroblast turnover could be an indicator of bronchial remodelling [48]. In vitro studies have demonstrated that smooth muscle cells infected with $C$. pneumoniae increase their MMP-1 and MMP-3 secretion [35], suggesting that this bacterial infection might favour airway remodelling. It may therefore be hypothesised that the pulmonary changes observed in Chl+ calves could be related to an increased pulmonary MMP-2 activity.

\subsection{Detection of chlamydiae in altered lung tissue}

Alterations of lung function, pulmonary lesions and the detection of markers of inflammation coincided well with PCR detection of chlamydiae in the Chl+ Group and the absence of the pathogen in the Chl- Group. Immunohistology was not sufficiently sensitive to detect chlamydial antigen in the pulmonary tissue of Chl+ calves. This is most likely due to the low level of chlamydial infection in subclinical cases. This further implies that immunohistology may fail to detect the agent even when pulmonary lesions are seen.

\subsection{Clinical outcome and pathogenesis of chlamydial infections within the respiratory system}

In previous studies on pigs, it became evident that respiratory infections with chlamydiae may vary from severe respiratory distress to mere colonisation without clinical or functional relevance within the respiratory system [32]. Data of the present study represent a third category of subclinical chlamydial infection characterised by persisting pulmonary inflammation and functional disorders of the peripheral respiratory system, but without clinical illness or macroscopic lesions of lung tissue. Although, at a first glance, such data seem to be conflicting, they were in good agreement with evidence from human medicine where the highest prevalence of respiratory chlamydial infections has been reported in children with recurrent or chronic bronchitis and pneumonia and in adults with COPD, indicating a higher prevalence from asymptomatic to severe, from upper to lower, and from acute to chronic respiratory tract involvement [40].

Data of this study indicate that the inapparent infections are not innocuous, but do cause minor inflammatory reactions. The clinical outcome itself may be influenced by host-bacteria interactions that include, on the one hand, (i) the chlamydial species involved, (ii) the number of chlamydiae present in the tissue, and (iii) the metabolic state of chlamydiae 
(activated or persistent), and, on the other hand, various factors of the host response. Interestingly, serological data might be related to the pathogenesis of chlamydial infections within the respiratory system. While a natural occurrence of chlamydiae in the respiratory tract was not associated with functional changes in the growing lungs until seven months of age in seronegative pigs, naturally acquired chlamydial infections in calves of the same age as presented here were associated with persisting antibody titres (data not shown) and pulmonary disorders. Also, the detection of $C$. pneumoniae in a carriage state (without serological response) was common with preference of lower respiratory tract disease in hospitalised children younger than six years having respiratory tract diseases [40].

In addition, several epidemiological risk factors, as well as co-infections, need to be taken into account with respect to the clinical outcome of chlamydial infections. In the presence of transport stress, climate change, or high-density host population, inapparent infections might build up to become clinically manifest ${ }^{1}$. Furthermore, it is likely that chlamydiae increase susceptibility to viral and bacterial co- or superinfection. From the pathogenetic point of view, $C$. pneumoniae is able to induce ciliary dysfunctions and epithelial damage in bronchial cells [43]. Consequently, impaired defence might facilitate adhesion of further micro-organisms to the bronchial system. In children with $C$. pneumoniae infection, bacterial co-infections and coinfections with mycoplasmas were identified as factors often associated with purulent bronchial inflammation and restrictive disturbances of lung function leading to more severe disease [42].

The calves examined in this study, however, were kept under standardised experimental conditions with respect to room climate, and there was no contact to other farm animals in order to avoid co- infections. Such conditions might explain the absence of clinical illness or remarkable clinical symptoms in the course of the study, although a higher respiratory index was seen in Chl+ calves compared to Chl- calves when calves were introduced in the premises indicating a higher presence of mild cough, nasal secretions and ocular secretions at the beginning of the study [33]. In contrast, Twomey et al. [53] described an outbreak of upper respiratory tract disease in young calves (aged four months old) characterised by pyrexia, mucous nasal discharge, conjunctivitis, and epiphora, and a morbidity rate of $100 \%$, where an aetiological role for Chlamydophila spp. was explored in field conditions.

\subsection{Influence of chlamydial infections on the functional maturation of the lung}

Based on the hypothesis that a naturally acquired chlamydial infection within the respiratory system would interfere with postnatal lung development, pulmonary functions were pursued over a period of 6 months in young calves aged 2 to 7 months. This is the period of postnatal lung growth and development which is determined by morphological differentiation and functional maturation of the lung. In calves approximately 30 days old, the bronchiolar epithelium is already well differentiated, and lung growth begins most rapidly at this age. In calves older than 30 days, there is an increase in alveolar number, and consequently in total alveolar surface area and in lung volume. In addition, mean bronchiolar cross-sectional area increases with age, and the percentage of terminal bronchioles decreases between one and 150 days of age [4]. In general, functional maturity of the respiratory system is not reached before one year of age or a body weight of $300 \mathrm{~kg}$ in the bovine species, and the growth of the 
airway and the alveolar structure seems to be non-isometric in large animals $[17,18]$. During this period, respiratory infections are likely involved in growth retardation that may cause impaired lung function for a long time and or even life long. Data of the present study allow the hypothesis that functional lung maturation could be restrained by a persisting chlamydial infection. This hypothesis, however, has yet to be corroborated by further studies.

\subsection{Conclusions}

In conclusion, naturally acquired respiratory infections with chlamydiae have been shown to be associated with pulmonary dysfunctions based on chronic, but clinically inapparent, inflammatory processes in the bovine lung during the period of functional maturation. The detection of both peripheral airway obstruction and pulmonary inflammation has quantitatively demonstrated the persisting effects of subclinical chlamydial infections on lung function. Because no animal model is available to examine the long-term effects of chronic respiratory chlamydial infections in vivo, data of the present study include a potential for modelling chlamydiaassociated chronic airway diseases (asthma and/or COPD) in a natural host of the chlamydial agent, rather than in rodent models which are more different in lung anatomy compared to humans and poorly suited for functional testing.

\section{ACKNOWLEDGEMENTS}

The authors are very grateful to Annelie Langenberg, Gerhard Foedisch, Sylke Stahlberg, and all colleagues of the technical staff of the animal house for their skilful assistance while performing the numerous lung function tests. They also thank Simone Bettermann, Christine Grajetzki, Sabine Lied and Monika Godat for excellent technical assistance and Wolfram Maginot for excellent photographic support. In addition, they are thankful to Dr Michael Rothe and Dr Gunter Becher (FILT GmbH, Berlin, Germany) for analysing concentrations of total protein and 8-IP. Help given by Dr H.-J. Zentis (Vet. Practitioner, Nideggen, Germany) in finding herds positive for chlamydial infections is very much appreciated. Furthermore, the authors wish to express their gratitude to Prof. Bernhard Kaltenboeck (Auburn University, AL, USA) for his critical reading of the manuscript.

\section{REFERENCES}

[1] Anderson M.L., Moore P.F., Hyde D.M., Dungworth D.L., Bronchus associated lymphoid tissue in the lungs of cattle: relationship to age, Res. Vet. Sci. (1986) 41:211220.

[2] Blasi F., Atypical pathogens and respiratory tract infections, Eur. Respir. J. (2004) 24:171-181.

[3] Branden E., Koyi H., Gnarpe J., Gnarpe H., Tornling G., Chronic Chlamydia pneumoniae infection is a risk factor for the development of COPD, Respir. Med. (2005) 99:20-26.

[4] Castleman W.L., Lay J.C., Morphometric and ultrastructural study of postnatal lung growth and development in calves, Am. J. Vet. Res. (1990) 51:789-795.

[5] Ehricht R., Slickers P., Goellner S., Hotzel H., Sachse K., Optimized DNA microarray assay allows detection and genotyping of single PCR-amplifiable target copies, Mol. Cell. Probes (2006) 20:60-63.

[6] Esposito S., Blasi F., Bellini F., Allegra L., Principi N., and the Mowgli Study Group, Mycoplasma pneumoniae and Chlamydia pneumoniae infections in children with pneumonia, Eur. Respir. J. (2001) 17:241245.

[7] Goellner S., Schubert E., Liebler-Tenorio E., Hotzel H., Saluz H.P., Sachse K., Transcriptional response patterns of Chlamydophila psittaci in different in vitro models of persistent infection, Infect. Immun. (2006) 74:4801-4808.

[8] Grayston J.T., Campbell L.A., Kuo C.C., Mordhorst C.H., Saikku P., Thom D.H.,Wang S.P., A new respiratory tract pathogen: Chlamydia pneumoniae strain TWAR, J. Infect. Dis. (1990) 161:618-625.

[9] Haas L.E., Tjan D.H., Schouten M.A., van Zanten A.R., Severe pneumonia from psittacosis in a bird-keeper, Ned. Tijdschr. Geneeskd. (2006) 150:117-121. 
[10] Hammerschlag M.R., Chlamydia pneumoniae and the lung, Eur. Respir. J. (2000) 16:1001-1007.

[11] Hogan R.J., Mathews S.A., Mukhopadhyay S., Summersgill J.T., Timms P., Chlamydial persistence: beyond the biphasic paradigm, Infect. Immun. (2004) 74:1843-1855.

[12] Janssen M.L., van de Wetering K., Arabin B., Sepsis due to gestational psittacosis: A multidisciplinary approach within a perinatological center - review of reported cases, Int. J. Fertil. Womens Med. (2006) 51:17-20.

[13] Jee J., DeGraves F.J., Kim T., Kaltenboeck B., High prevalence of natural Chlamydophila spp. infection in calves, J. Clin. Microbiol. (2004) 42:5664-5672.

[14] Johnston S.L., Martin R.J., Chlamydophila pneumoniae and Mycoplasma pneumoniae - A role in asthma pathogenesis? Am. J. Respir. Crit. Care Med. (2005) 172:10781089.

[15] Kirschvink N., Vincke G., Fievez L., Onclinx C., Wirth D., Cataldo D., Louis R., Peck M., Gustin P., Repeated cadmium nebulizations induce pulmonary MMP-2 and MMP-9 production and emphysema in rats, Toxicology (2005) 211:36-48.

[16] Lakritz J., Marsh A., Cockrell M., Smith M.F., Tyler J.W., Characterization of gelatinases in bronchoalveolar labage fluid and gelatinases produced by alveolar macrophages isolated from healthy calves, Am. J. Vet. Res. (2004) 65:163-172.

[17] Lekeux P., Hajer R., Breukink H.J., Effect of somatic growth on pulmonary function values in healthy Friesian cattle, Am. J. Vet. Res. (1984) 45:2003-2007.

[18] Lekeux P., Pulmonary function in healthy, exercising and diseased animals. Vlaams Diergegeneeskunding Tijdschrift 1993, Universiteit Gent, Belgium, special issue, 1993.

[19] Littman A.J., Jackson L.A., Vaughan T.L., Chlamydia pneumoniae and lung cancer: epidemiologic evidence, Cancer Epidemiol. Biomarkers Prev. (2005) 14:773-778.

[20] Livingstone M., Longbottom D., What is the prevalence and economic impact of chlamydial infections in cattle? The need to validate and harmonise existing methods of detection, Vet. J. (2006) 172:3-5.

[21] Marrie T.J., Peeling R.W., Reid T., De Carolis E., and the Canadian CommunityAcquired Pneumonia Investigators, Chlamydia species as a cause of communityacquired pneumonia in Canada, Eur. Respir. J. (2003) 21:779-784.

[22] Martin R.J., Infections and asthma, Clin. Chest Med. (2006) 27:87-98.
[23] Mead J., Mechanical properties of the lung, Physiol. Rev. (1961) 41:281-329.

[24] Mitzner W., Collateral ventilation, in: Crystal R.G., West J.B., et al. (Eds.), The Lung, Scientific Foundations, Raven Press, New York, 1991, pp. 1053-1063.

[25] Mpiga P., Ravaoarinoro M., Chlamydia trachomatis persistence: an update, Microbiol. Res. (2006) 161:9-19.

[26] Normann E., Gnarpe J., Wettergren B., Janson C., Wickman M., Nordvall L., The association between Chlamydia pneumoniae antibodies and wheezing in young children and the influence of sex, Thorax (2006) 61:1054-1058.

[27] Otter A., Twomey D.F., Rowe N.S., Tipp J.W., McElligott W.S., Griffiths P.C., O’Neill P., Suspected chlamydial keratoconjunctivitis in British cattle, Vet. Rec. (2003) 152:787-788

[28] Pandeli V., Ernest D., A case of fulminant psittacosis, Crit. Care Resusc. (2006) 8:40-42.

[29] Reinhold P., MacLeod D., Lekeux P., Comparative evaluation of impulse oscillometry and a monofrequency forced oscillation technique in clinically healthy calves undergoing bronchochallenges, Res. Vet. Sci. (1996) 61:206-213.

[30] Reinhold P., Smith H.-J., Langenberg A., Lekeux P., Measurement of respiratory impedance in healthy calves using the impulse oscillation technique - physiological and methodological aspects, Vet. J. (1998) 155:27-38.

[31] Reinhold P., Smith H.-J., Close R., Genicot B., Lekeux P., Validation of impulse oscillometry in Friesian and Blue Belgian Calves with respect to changes in extrathoracic upper airway resistance, Res. Vet. Sci. (1998) 65:93-102.

[32] Reinhold P., Jaeger J., Melzer F., Sachse K., Evaluation of lung function in pigs either experimentally or naturally infected with Chlamydiaceae, Vet. Res. Commun. (2005) (Suppl.1) 29:125-150.

[33] Reinhold P., Jaeger J., Liebler-Tenorio E., Berndt A., Bachmann R., Schubert E., Melzer F., Elschner M., Sachse K., Impact of latent infections with Chlamydophila species in young cattle, Vet. J. (2007) in press, doi: 10.1016/j.tvj1.2007.01.004 (E-pub).

[34] Robinson N.E., Some functional consequences of species differences in lung anatomy, Adv. Vet. Sci. Comp. Med. (1982) 26:1-33.

[35] Rödel J., Prochnau D., Prager K., Pentcheva E., Hartmann M., Straube E., Increased production of matrix metalloproteinases 1 and 3 
by smooth muscle cells upon infection with Chlamydia pneumoniae, FEMS Immunol. Med. Microbiol. (2003) 38:159-164.

[36] Sachse K., Hotzel H., Detection and differentiation of chlamydiae by nested PCR, in: Sachse K., Frey J. (Eds.), PCR Detection of Microbial Pathogens, Humana Press, Totowa, NJ, USA, 2002, pp. 123-136.

[37] Sachse K., Hotzel H., Slickers P., Ellinger T., Ehricht R., DNA microarray based detection and identification of Chlamydia and Chlamydophila, Mol. Cell. Probes (2005) 19:41-50.

[38] Saikku P., The epidemiology and significance of Chlamydia pneumoniae, J. Infect. Dis. (1992) 25:27-34.

[39] Schmidt S.M., Müller C.E., Bruns R., Wiersbitzky S.K.W., Bronchial Chlamydia pneumoniae infection, markers of allergic inflammation and lung function in children, Pediatr. Allergy Immunol. (2001) 12:257265.

[40] Schmidt S.M., Müller C.E., Krechting M., Wiersbitzky H., Gürtler L., Wiersbitzky S.K.W., Chlamydia pneumoniae carriage and infection in hospitalized children with respiratory tract diseases, Infection (2003) 31:410-416.

[41] Schmidt S.M., Müller C.E., Wiersbitzky S.K.W., Inverse association between Chlamydia pneumoniae respiratory tract infection and initiation of asthma or allergic rhinitis in children, Pediatr. Allergy Immunol. (2005) 16:137-144.

[42] Schmidt S.M., Müller C.E., Gürtler L., Bruns R., Ballke E.H., Wiersbitzky H., Ehlers M., Rose H.J., Wiersbitzky S.K.W., Chlamydophila pneumoniae respiratory tract infection aggravates therapy refractory bronchitis or pneumonia in childhood, Klin. Pädiatr. (2005) 217:9-14.

[43] Shemer-Avni Y., Liebermann D., Chlamydia pneumoniae-induced ciliostasis in ciliated bronchial epithelial cells, J. Infect. Dis. (1995) 171:1274-1278.

[44] Simonen-Jokinen T.L.M., Eskelinen U.M., Härtel H.M., Nikunen S.K., Saloniemi H.S., Maisi P.S., Gelatinolytic matrix metalloproteinases-2 and -9 in tracheobronchial lavage fluid obtained from calves with concurrent infections of Pasteurella multocida and Mycoplasma bovirhinis, Am. J. Vet. Res. (2005) 66:2101-2106.

[45] Smith H.-J., Reinhold P., Goldman M.D., Forced oscillation technique and impulse oscillometry, Eur. Respir. Monogr. (2005) 31:72-105.

[46] Stephens R.S., The cellular paradigm of chlamydial pathogenesis, Trends Microbiol. (2003) 11:44-51.
[47] Storz J., Kaltenboeck B., Diversity of chlamydia-induced diseases, in: Woldehiwet Z., Ristic M. (Eds.), Rickettsial and chlamydial diseases of domestic animals, Pergamon Press, Oxford/UK, 1993, pp. 363-393.

[48] Suzuki R., Miyazaki Y., Takagi K., Torii K., Taniguchi H., Matrix metalloproteinases in the pathogenesis of asthma and COPD: implications for therapy, Treat. Respir. Med. (2004) 3:17-27.

[49] ten Brinke A., van Dissel J.T., Sterk P.J., Zwinderman A.H., Rabe K.F., Bel E.H., Persistent airflow limitation in adult-onset nonatopic asthma is associated with serologic evidence of Chlamydia pneumoniae infection, J. Allergy Clin. Immunol. (2001) 107:449-454.

[50] Theegarten D., Mogilevski G., Anhenn O., Stamatis G., Jaeschock R., Morgenroth K., The role of Chlamydia in the pathogenesis of pulmonary emphysema. Electron microscopy and immunofluorescence reveal corresponding findings as in atherosclerosis, Virchows Arch. (2000) 437:190-193.

[51] Theegarten D., Anhenn O., Hotzel H., Wagner M., Marra A., Stamatis G., Mogilevski G., Sachse K., A comparative ultrastructural and molecular biological study on Chlamydia psittaci infection in alpha-1 antitrypsin deficiency and nonalpha-1 antitrypsin deficiency emphysema versus lung tissue of patients with hamartochondroma, BMC Infect. Dis. (2004) $4: 38$.

[52] Twomey D.F., Griffiths P.C., Hignett B.C., Martin T.P., Suspected chlamydial polyarthritis in a calf in the UK, Vet. Rec. (2003) 152:340.

[53] Twomey D.F., Griffiths P.C., Horigan M.W., Hignett B.C., Martin T.P., An investigation into the role of Chlamydophila spp. in bovine upper respiratory tract disease, Vet. J. (2006) 171:574-576.

[54] Walder G., Schonherr H., Hotzel H., Speth C., Oehme A., Dierich M.P., Wurzner R., Presence of Chlamydophila psittaci DNA in the central nervous system of a patient with status epilepticus, Scand. J. Infect. Dis. (2003) 35:71-73.

[55] Walder G., Hotzel H., Brezinka C., Gritsch W., Tauber R., Wurzner R., Ploner F., An unusual cause of sepsis during pregnancy: recognizing infection with Chlamydophila abortus, Obstet. Gynecol. (2005) 106:12151217.

[56] Webley W.C., Salva P.S., Andrzejewski C., Cirino F., West C.A., Tilahun Y., Stuart E.S., The bronchial lavage of pediatric patients with asthma contains infectious Chlamydia, Am. J. Respir. Crit. Care Med. (2005) 171:1083-1088. 